\title{
Complex Descartes Circle Theorem
}

\author{
Sam Northshield
}

\begin{abstract}
We present a short proof of Descartes Circle Theorem on the "curvaturecenters" of four mutually tangent circles. Key to the proof is associating an octahedral configuration of spheres to four mutually tangent circles. We also prove an analogue for spheres.
\end{abstract}

It can be traced back to at least Descartes that four mutually tangent circles have curvatures (reciprocals of radii) satisfying the relation

$$
(a+b+c+d)^{2}=2\left(a^{2}+b^{2}+c^{2}+d^{2}\right) .
$$

The Monthly has published several papers concerning this fascinating topic: $[1,3,4,5,6]$. It was only in 2001 [3] that is was noticed, and proved, that the "curvature-centers" (curvature times center where the center is considered a complex number) satisfy the same relation. We present a short proof of this result (Theorem 1) and an analogous version for spheres (Corollary 1).

For the purposes of this paper, a sphere will alway be contained in the halfspace $\mathbb{C} \times[0, \infty)$ and be tangent to the complex plane. Let $S(z, r)$ denote the sphere with radius $r$ tangent to $\mathbb{C}$ at $z$. It is obvious that $S(z, r)$ and $S(w, s)$ are tangent to each other if and only if

$$
|z-w|^{2}=4 r s .
$$

It is also immediate that given any three points $z_{1}, z_{2}, z_{3} \in \mathbb{C}$, there are unique numbers $r_{1}, r_{2}, r_{3}$ such that the spheres $S\left(z_{i}, r_{i}\right)$ are mutually tangent. In particular, if $\{i, j, k\}=\{1,2,3\}$ then

$$
r_{i}=\frac{\left|z_{i}-z_{j}\right| \cdot\left|z_{i}-z_{k}\right|}{2\left|z_{j}-z_{k}\right|}
$$

We say that two circles are orthogonal if they intersect at right angles; see Figure 1.

Lemma 1. Let $C_{1}, C_{2}$ be two orthogonal circles which intersect at $w_{1}, w_{2}$ and that have curvatures $c_{1}, c_{2}$ and centers $z_{1}, z_{2}$ respectively. Let $k_{1}, k_{2}$ be the curvatures of any two tangent spheres tangent to $\mathbb{C}$ at $w_{1}, w_{2}$ respectively. Then

$$
k_{1} k_{2}=\frac{4}{\left|w_{1}-w_{2}\right|^{2}}=c_{1}^{2}+c_{2}^{2}
$$


and

$$
k_{1} k_{2} w_{1} w_{2}=\frac{4 w_{1} w_{2}}{\left|w_{1}-w_{2}\right|^{2}}=c_{1}^{2} z_{1}^{2}+c_{2}^{2} z_{2}^{2} .
$$

Proof. Let $S(w, r)$ be any sphere tangent to both $S\left(w_{1}, r_{1}\right)$ and $S\left(w_{2}, r_{2}\right)$ where $r_{i}=1 / k_{i}$ for $i=1,2$. Then, by $(2)$,

$$
k_{1} k_{2}=\frac{2\left|w_{2}-w\right|}{\left|w_{2}-w_{1}\right|\left|w-w_{1}\right|} \frac{2\left|w_{1}-w\right|}{\left|w_{2}-w_{1}\right|\left|w-w_{2}\right|}=\frac{4}{\left|w_{1}-w_{2}\right|^{2}} .
$$

The quadrilateral $\left[z_{1}, w_{1}, z_{2}, w_{2}\right]$ in Figure 1 has area represented both as $r_{1} r_{2}$ and as $\left|z_{1}-z_{2}\right|\left|w_{1}-w_{2}\right| / 2$. Hence,

$$
c_{1}^{2}+c_{2}^{2}=\frac{r_{1}^{2}+r_{2}^{2}}{\left(r_{1} r_{2}\right)^{2}}=\frac{\left|z_{1}-z_{2}\right|^{2}}{\left|z_{1}-z_{2}\right|^{2}\left|w_{1}-w_{2}\right|^{2} / 4}=\frac{4}{\left|w_{1}-w_{2}\right|^{2}}
$$

and so (a) is shown.

Without loss of generality, $z_{2}-z_{1}, i\left(w_{2}-w_{1}\right) \in \mathbb{R}$. Let $z$ and $a$ be as labelled in Figure 1. In particular, let $a$ denote half the distance between $w_{1}$ and $w_{2}$. Then, by part (a),

$$
a^{2}=\left|w_{1}-w_{2}\right|^{2} / 4=1 /\left(k_{1} k_{2}\right)=1 /\left(c_{1}^{2}+c_{2}^{2}\right) .
$$

Referring again to Figure 1,

$$
z_{1}=z-\sqrt{r_{1}^{2}-a^{2}}, z_{2}=z+\sqrt{r_{2}^{2}-a^{2}}, w_{1}=z+i a, w_{2}=z-i a,
$$

and so, using $c_{1}=1 / r_{1}$ and equation (3),

$$
c_{1}^{2} \sqrt{r_{1}^{2}-a^{2}}=c_{1} \sqrt{1-a^{2} c_{1}^{2}}=\frac{c_{1} c_{2}}{\sqrt{k_{1} k_{2}}}=c_{2}^{2} \sqrt{r_{2}^{2}-a^{2}} .
$$

Hence, using (4),

$$
\begin{aligned}
& c_{1}^{2} z_{1}^{2}=c_{1}^{2} z^{2}-2 z c_{1}^{2} \sqrt{r_{1}^{2}-a^{2}}+1-c_{1}^{2} a^{2}, \\
& c_{2}^{2} z_{2}^{2}=c_{2}^{2} z^{2}+2 z c_{2}^{2} \sqrt{r_{2}^{2}-a^{2}}+1-c_{2}^{2} a^{2}
\end{aligned}
$$

and thus

$$
c_{1}^{2} z_{1}^{2}+c_{2}^{2} z_{2}^{2}=\left(c_{1}^{2}+c_{2}^{2}\right) z^{2}+1=k_{1} k_{2}\left(z^{2}+a^{2}\right)=k_{1} k_{2} w_{1} w_{2}
$$

which shows part (b).

Lemma 2. Given three mutually tangent circles $C_{1}, C_{2}, C_{3}$ with curvatures $c_{1}, c_{2}, c_{3}$ and respective centers $z_{1}, z_{2}, z_{3}$, let $c$ and $z$ be the curvature and center respectively of the circle orthogonal to each of the three given circles. For $i \neq j \in\{1,2,3\}$, let $\left\{z_{i j}\right\}=C_{i} \cap C_{j}$, let $S_{i j}$ denote the unique sphere tangent 


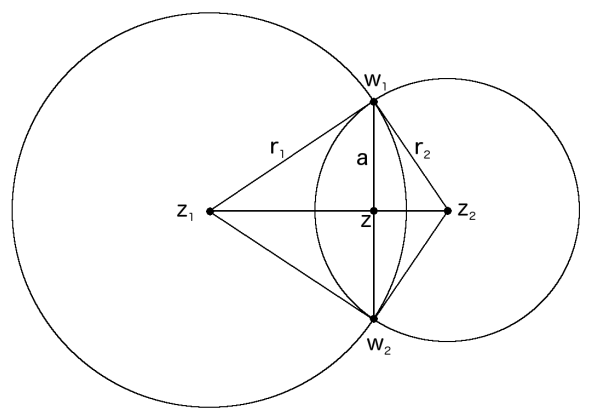

Figure 1: Two Orthogonal Circles

at $z_{i j}$ such that all three spheres $S_{12}, S_{13}, S_{23}$ are mutually tangent, and let $k_{i j}$ denote curvature of $S_{i j}$. Then

$k_{i j}=c_{i}+c_{j}$, and

$$
c^{2} z^{2}=c_{1} z_{1} c_{2} z_{2}+c_{1} z_{1} c_{3} z_{3}+c_{2} z_{2} c_{3} z_{3} .
$$

Proof. By Coxeter [2, p. 15], $c=\sqrt{c_{1} c_{2}+c_{1} c_{3}+c_{2} c_{3}}$. By Lemma 1,

$$
c^{2}+c_{1}^{2}=k_{12} k_{13}, c^{2}+c_{2}^{2}=k_{12} k_{23}, c^{2}+c_{3}^{2}=k_{13} k_{23},
$$

and so $k_{i j}=c_{i}+c_{j}$ and part (a) is shown.

Given two tangent circles with centers $z, w$ and respective radii $r, s$, it is easy to see that the tangent point is $(s z+r w) /(r+s)$. Hence,

$$
z_{i j}=\frac{c_{i} z_{i}+c_{j} z_{j}}{c_{i}+c_{j}}
$$

and so, by part (a),

$$
k_{i j} z_{i j}=c_{i} z_{i}+c_{j} z_{j}
$$

By Lemma 1b,

$$
c^{2} z^{2}+c_{3}^{2} z_{3}^{2}=k_{13} k_{23} z_{13} z_{23}=\left(c_{1} z_{1}+c_{3} z_{3}\right)\left(c_{2} z_{2}+c_{3} z_{3}\right)
$$

and therefore

$$
c^{2} z^{2}=c_{1} z_{1} c_{2} z_{2}+c_{1} z_{1} c_{3} z_{3}+c_{2} z_{2} c_{3} z_{3}
$$

which shows part (b).

Given four mutually tangent circles, there are six points of tangency between them (see Figure 2). At each such point $z$, assign a sphere tangent to the plane at $z$ with curvature equal to the sum of the curvatures of the two circles that 
meet at $z$. By Lemma 2(a), the six spheres have disjoint interiors and any two are tangent to each other if and only if their points of tangency to the plane are on the same circle. We will refer to this collection of spheres as the octahedral arrangement of spheres associated with the four circles since the adjacency graph of these six circles forms an octahedron.

A key idea for the proof of the main theorem is that the respective octahedral arrangements of spheres associated to $\left\{C_{i}\right\}$ and to $\left\{C_{i}^{\prime}\right\}$ are the same. We now show the Complex Descartes Circle Theorem.

Theorem 1. Given four mutually tangent circles with curvatures $c_{i}$ and respective centers $z_{i}$,

$$
\left(\sum c_{i} z_{i}\right)^{2}=2 \sum c_{i}^{2} z_{i}^{2}
$$

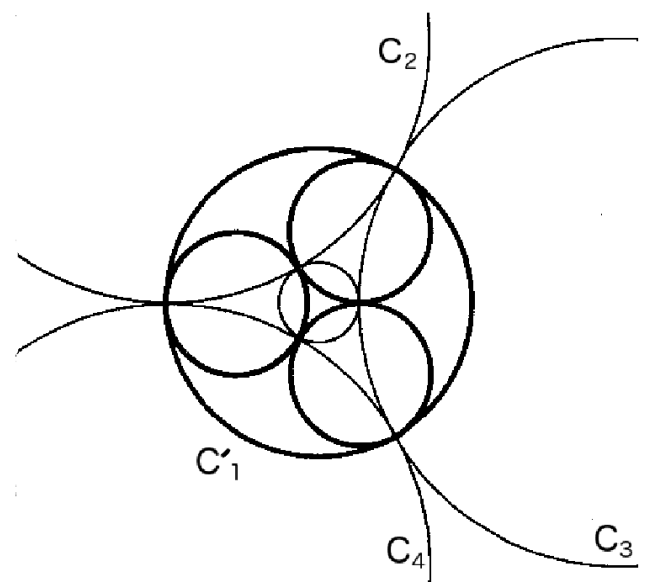

Figure 2: Four circles and their duals; three circles and their dual are labelled.

Proof. Consider a configuration of four mutually tangent circles $C_{1}, C_{2}, C_{3}, C_{4}$. We define $C_{1}^{\prime}$ to be the circle containing the three tangency points of $C_{2}, C_{3}, C_{4}$, we define $C_{2}^{\prime}, C_{3}^{\prime}, C_{4}^{\prime}$ similarly, and we let $c_{i}^{\prime}$ denote the curvature of $C_{i}^{\prime}$. These form what is called the dual configuration, see Figure 2, where $C_{1}^{\prime}, C_{2}, C_{3}$ and $C_{4}$ are labelled.

For $i \neq j \in\{1,2,3,4\}$, let $S_{i j}$ be the sphere tangent to the plane at $C_{i} \cap C_{j}$ with curvature $k_{i j}:=c_{i}+c_{j}$. By Lemma $2(\mathrm{a})$, the spheres $\left\{S_{i j}: 1 \leq i<j \leq 4\right\}$ form the octahedral arrangement of spheres associated with the circles $C_{1}, \ldots, C_{4}$. Similarly, let $S_{i j}^{\prime}$ be the sphere tangent to the plane at $C_{i}^{\prime} \cap C_{j}^{\prime}$ with curvature $k_{i j}^{\prime}:=c_{i}^{\prime}+c_{j}^{\prime}$.

If $\{i, j, m, n\}=\{1,2,3,4\}$, then $S_{i j}=S_{m n}^{\prime}$ and thus $k_{i j}=k_{m n}^{\prime}$. By (5), $z_{i j}^{\prime}=z_{m n}$ and thus

$$
c_{i}^{\prime} z_{i}^{\prime}+c_{j}^{\prime} z_{j}^{\prime}=\left(c_{i}^{\prime}+c_{j}^{\prime}\right) z_{i j}^{\prime}=\left(c_{m}+c_{n}\right) z_{m n}=c_{m} z_{m}+c_{n} z_{m} .
$$


For convenience, let $w_{i}=c_{i} z_{i}, w_{i}^{\prime}=c_{i}^{\prime} z_{i}^{\prime}, K_{i j}=k_{i j} z_{i j}$, and $K_{i j}^{\prime}=k_{i j}^{\prime} z_{i j}^{\prime}$. By Lemma $2(\mathrm{~b}), w_{4}^{\prime}=\sigma \sqrt{w_{1} w_{2}+w_{1} w_{3}+w_{2} w_{3}}$ (where $\sigma$ is either 1 or -1 ) since $C_{4}^{\prime}$ is the incircle of the triangle connecting the centers of $C_{1}, C_{2}$, and $C_{3}$. Hence

$$
\begin{aligned}
2\left(w_{1}+w_{2}+w_{3}-w_{4}\right) & =K_{12}+K_{34}+K_{12}+K_{13}+K_{23}-K_{14}-K_{24}-K_{34} \\
& =K_{34}^{\prime}+K_{12}^{\prime}+K_{34}^{\prime}+K_{24}^{\prime}+K_{14}^{\prime}-K_{23}^{\prime}-K_{13}^{\prime}-K_{12}^{\prime} \\
& =4 w_{4}^{\prime}=4 \sigma \sqrt{w_{1} w_{2}+w_{1} w_{3}+w_{2} w_{3}} .
\end{aligned}
$$

It follows that

$$
w_{4}=w_{1}+w_{2}+w_{3}+2 \sigma \sqrt{w_{1} w_{2}+w_{1} w_{3}+w_{2} w_{3}}
$$

and the result follows from the fact that equation (1) is equivalent to

$$
d=a+b+c \pm 2 \sqrt{a b+a c+b c} .
$$

The Complex Descartes Circle Theorem is a true generalization of the Descartes Circle Theorem since replacing $z_{i}$ by $\left(z_{i}+z\right) / z$ in the formula and taking the limit as $z$ goes to infinity gives the old version.

The spheres in an octahedral arrangement associated with four mutually tangent circles obeys a similar formula (the proof of which follows immediately from Theorem 1).

Corollary 1. Given four mutually tangent circles $C_{1}, C_{2}, C_{3}, C_{4}$, and $i \neq j \in$ $\{1,2,3,4\}$, let $\left\{z_{i j}\right\}=C_{i} \cap C_{j}, S_{i j}$ be the sphere tangent to plane at $z_{i j}$ with curvature $k_{i j}:=c_{i}+c_{j}$. Then,

$$
2\left(\sum_{i<j} k_{i j}\right)^{2}=9 \sum_{i<j} k_{i j}^{2}
$$

$$
2\left(\sum_{i<j} k_{i j} z_{i j}\right)^{2}=9 \sum_{i<j} k_{i j}^{2} z_{i j}^{2}
$$

\section{References}

[1] H.S.M. Coxeter, The Problem of Apollonius, Amer. Math. Monthly 75 (1968) 5-15.

[2] H.S.M. Coxeter, Introduction to Geometry, second edition, John Wiley and Sons, New York, 1969.

[3] J. Lagarias, C. Mallows, A. Wilks, Beyond the Descartes circle theorem, Amer. Math. Monthly 109 (2002) 338-361. 
[4] D. Pedoe, On a Theorem in geometry, Amer. Math. Monthly 74 (1967) 627-640.

[5] P. Sarnak, Integral Apollonian Packings, Amer. Math. Monthly 118 (2011) 291-306.

[6] J.B. Wilker, Four Proofs of a Generalization of the Descartes Circle Theorem, Amer. Math. Monthly 76 (1969) 278-282.

Department of Mathematics, SUNY, Plattsburgh, NY 12901

northssw@plattsburgh.edu 